\title{
Artificially Structured Boundary for a High Purity Ion Trap or Ion Source
}

\author{
J. L. Pacheco, C. A. Ordonez, and D. L. Weathers \\ Department of Physics, University of North Texas, Denton, Texas 76203
}

\begin{abstract}
A plasma enclosed by an Artificially Structured Boundary (ASB) is proposed here as an alternative to existing ion source assemblies. In accelerator applications, many ion sources can have a limited lifetime or frequent service intervals due to sputtering and eventual degradation of the ion source assembly. Ions are accelerated towards the exit canal of positive ion sources, whereas, due to the biasing scheme, electrons or negative ions are accelerated towards the back of the ion source assembly. This can either adversely affect the experiment in progress due to sputtered contamination or compromise the integrity of the ion source assembly. Charged particle trajectories in the proximity of an ASB experience electromagnetic fields that are designed to hinder ion-surface interactions. Away from the ASB there is an essentially field free region. The field produced by an ASB is considered to consist of a periodic sequence of electrostatically plugged magnetic field cusps. A classical trajectory Monte Carlo simulation is extended to include electrostatic plugging of magnetic field cusps. The conditions necessary for charged particles to be reflected by the ASB are presented and quantified in terms of normalized parameters.
\end{abstract}

Keywords: Ion Source, Ion Trap, Artificially Structured Boundary 


\section{INTRODUCTION}

In the application of ion sources for accelerator physics, plasma physics, or plasma processing purposes, a clean source of ions is desirable when unwanted sputtered contamination is detrimental for the experiment at hand or for the ion source assembly itself. In addition, in the accumulation of rare species of ions or antimatter particles, good confinement is particularly important due to the limited availability of the particles being trapped. Ions within an ion source that impinge on a surrounding material surface can alter the physical properties of the surface. Furthermore, ion sources that employ reactive metals can require frequent service intervals. The study presented here proposes a configuration that can minimize the interaction of a plasma with material surfaces.

An Artificially Structured Boundary (ASB) is described here as a material boundary that produces electrostatic and magnetostatic fields for the purpose of modifying charged particle trajectories when charged particles approach the boundary. Such an ASB is considered here to form a periodic set of cusping magnetic fields with electrostatic potential barriers at the location of the magnetic field cusps. An ASB that produces purely magnetic fields, without the electrostatic barriers, is described in [1]. Two properties of such an arrangement are notable: (1) The ASB is capable of simultaneously reflecting charged particles of either sign of charge, but only when the particles are incident at shallow angles. (2) A nearly field free region occurs away from the ASB so that the field only modifies charged particle trajectories close to the material boundary. Charged particle trajectories that are normal to the ASB can escape through magnetic field cusps if no electrostatic plugging is present. Preliminary experimental research has been reported in which a plasma interacts with a segment of an ASB with electrostatic plugging [2]. Also, theoretical research has been reported on possible applications of an ASB for lining an electrostatic storage ring [3] and for bounding a confined plasma [4]. The current study assesses the effect that incorporating electrostatic plugging of the magnetic field cusps can have on the confinement of charged particles of a single sign of charge. The configuration may serve to confine a two-species plasma, with the first species confined by the ASB and the second, oppositely signed species, confined by the space charge of the first species [4].

In Sec. II, the fields employed for confinement are described. A normalization scheme is developed in Sec. II A and normalized equations of motion are derived in Sec. II B. The 
method of solution is presented in Sec. II C. Results are presented in Sec. III. Concluding remarks are presented in Sec. IV.

\section{THEORY}

The current study considers the interaction of a single charged particle with an ASB. The effects due to the collective nature of plasmas are not taken into account here. Charged particle trajectories near an ASB are determined by solving Newton's second law. Figure 1 depicts the characteristics of the simulation environment.

The magnetic field developed in [5] is used here, except that (1) the magnetic field dependence on the coordinates is changed, and (2) the strength of the field is defined by the conditions necessary for magnetic confinement. Such a field has the form [5]

$$
\boldsymbol{B}(x, y, z)=B_{0} \boldsymbol{\beta}_{t}\left(\frac{x}{S}, \frac{y}{S}, \frac{z}{S}\right)
$$

with

$$
\boldsymbol{\beta}_{t}\left(x_{n}, y_{n}, z_{n}\right)=\sum_{i=1}^{N} \eta_{i} \boldsymbol{\beta}\left(x_{n}, y_{n}-(i-1) \Delta y_{n}, 0\right)
$$

and

$$
\boldsymbol{\beta}\left(x_{n}, y_{n}, z_{n}\right)=\frac{-\cos \left(2 \pi x_{n}\right) \sinh \left(2 \pi y_{n}\right)}{\cos \left(4 \pi x_{n}\right)-\cosh \left(4 \pi y_{n}\right)} \hat{\boldsymbol{x}}+\frac{\sin \left(2 \pi x_{n}\right) \cosh \left(2 \pi y_{n}\right)}{\cos \left(4 \pi x_{n}\right)-\cosh \left(4 \pi y_{n}\right)} \hat{\boldsymbol{y}} .
$$

Here $\boldsymbol{r}_{n}=x_{n} \hat{\boldsymbol{x}}+y_{n} \hat{\boldsymbol{y}}+z_{n} \hat{\boldsymbol{z}}=\frac{\boldsymbol{r}}{S} ; \boldsymbol{\beta}\left(x_{n}, y_{n}, z_{n}\right)$ describes the direction of the magnetic field created by a planar array of current carrying wires that has a spatial period $S$, that is infinite in $z$ dimension and coincides with the $y=0$ plane; and $\eta_{i}$ assigns relative current factors for each of the $N(=10)$ planar arrays that are stacked $\Delta y_{n}$ apart. $\eta_{1}=\eta_{10}=1.27$ and $\eta_{i}=1$ for $2 \leq i \leq 9$. $B_{0}$ is approximately equal to the magnitude of the magnetic field at the center of the anode gap. An expression for $B_{0}$ will be developed in Sec. II A.

The electric field used for electrostatic plugging of the magnetic field cusps is obtained by numerically computing the electrostatic potential $\phi(x, y, z)$ and then using $\boldsymbol{E}(x, y, z)=$ $-\nabla \phi(x, y, z)$. The numerical computation of $\phi$ is described in Sec. II C.

\section{A. Normalization}

Consider a collisionless, non-drifting, unmagnetized plasma that follows a Maxwellian velocity distribution. From this point onward, an ensemble of charged particles is loosely 
referred to as a plasma. $T$ is the temperature, in units of energy, associated with the Maxwellian distribution and $m$ is the mass of a plasma particle. Assume that the plasma is composed of a single species of charged particles, each of which has a positive charge $q$ (e.g., $q=2 e$ for a doubly ionized positive species, and $e$ is the electronic charge). In what follows, the quantities $m, q, S$, and $3 T / 2$ are employed to carry out a normalization procedure. $S$ is the spatial period of the magnetic field, and $3 T / 2$ is the average kinetic energy per particle in a Maxwellian source of particles. The normalized parameters are $t_{n}=\frac{t}{S} \sqrt{\frac{3 T}{2 m}}$, $\boldsymbol{v}_{n}=\boldsymbol{v} \sqrt{\frac{2 m}{3 T}}, \boldsymbol{r}_{n}=\frac{\boldsymbol{r}}{S}, \boldsymbol{a}_{n}=\boldsymbol{a} \frac{2 m S}{3 T}, \boldsymbol{B}_{n}=\boldsymbol{B} S q \sqrt{\frac{2}{3 m T}}, \boldsymbol{E}_{n}=\boldsymbol{E} \frac{2 S q}{3 T}$, and $\phi_{n}=\frac{2 q \phi}{3 T}$, which are the dimensionless normalized time, position, velocity, acceleration, magnetic field, electric field, and electric potential, respectively. Newton's second law for a charged particle that experiences a Lorentz force is

$$
\boldsymbol{a}_{n}=\boldsymbol{E}_{n}+\left(\boldsymbol{v}_{n} \times \boldsymbol{B}_{n}\right)
$$

when written in terms of the normalized parameters. The normalized electric field is

$$
\boldsymbol{E}_{n}=-\nabla \phi_{n}\left(x_{n}, y_{n}, z_{n}\right)
$$

Consider a positive charged particle in a region with electrostatic potential $\phi$, which is positive or zero everywhere accessible to the particle. In particular, consider the electrostatic potential present in the ASB described in Fig. 1, where $\phi=\phi_{0}$ at the center of the anode gap between electrodes labeled by $V_{1}$. The electrostatic potential energy barrier, $U_{0}=q \phi_{0}$, reflects charged particles that start at zero potential with less kinetic energy than is required to overcome the potential energy barrier. Define the ratio of the electrostatic potential energy barrier, at the location of $\phi_{0}$, to the average kinetic energy of a plasma particle to be the normalized potential barrier,

$$
\phi_{n 0}=\frac{2 q \phi_{0}}{3 T}
$$

The Larmor radius, $R_{\mathrm{L}}$, is used to specify a condition for magnetic confinement. At the center of the anode gap, the magnetic field has a magnitude specified by $B_{0}$, so that $R_{\mathrm{L}}=\frac{m v_{\mathrm{p}}}{q B_{0}}$. Here $v_{\mathrm{p}}$ is the magnitude of the velocity component perpendicular to the direction of the magnetic field at the location of $B_{0}$. In order for a charged particle to experience magnetic confinement in the anode gap, its Larmor radius must be much smaller than the space between two adjacent columns of wires, i.e. $R_{\mathrm{L}} \ll \frac{S}{4}$. Let the average thermal energy be available to a plasma particle's motion perpendicular to the magnetic field. In such case 
$\frac{3}{2} T=\frac{m}{2} v_{\mathrm{p}}^{2}$, which leads to

$$
B_{0}=\frac{\sqrt{3 m T}}{q R_{\mathrm{L}}} .
$$

With the magnetic field given by Eq. (1), and defining an inverse normalized Larmor radius $\delta=\frac{S}{R_{\mathrm{L}}}$, the normalized magnetic field becomes

$$
\boldsymbol{B}_{n}=\sqrt{2} \delta \boldsymbol{\beta}_{t}\left(x_{n}, y_{n}, z_{n}\right)
$$

where $\delta \gg 4$ is considered necessary for magnetic confinement.

\section{B. Equations of Motion}

The equations of motion are obtained from Eqs. (4), (5), and (8). For the planar system considered in the current study, the fields are completely independent of the $z$-coordinate. Therefore, the equations of motion become

$$
\begin{aligned}
& x_{n}^{\prime \prime}\left(t_{n}\right)=E_{n x}-\sqrt{2} \delta\left(z_{n}^{\prime}\left(t_{n}\right) \beta_{t y}\right), \\
& y_{n}^{\prime \prime}\left(t_{n}\right)=E_{n y}+\sqrt{2} \delta\left(z_{n}^{\prime}\left(t_{n}\right) \beta_{t x}\right),
\end{aligned}
$$

and

$$
z_{n}^{\prime \prime}\left(t_{n}\right)=\sqrt{2} \delta\left(x_{n}^{\prime}\left(t_{n}\right) \beta_{t y}-y_{n}^{\prime}\left(t_{n}\right) \beta_{t x}\right)
$$

Here, $\beta_{t x}, E_{n x}$ and $\beta_{t y}, E_{n y}$ are the $x$ and $y$ components of $\boldsymbol{\beta}_{t}$ and $\boldsymbol{E}_{n}$, respectively. The equations of motion (Eqs. (9)-(11)) were solved simultaneously to obtain parametric trajectories in three dimensions.

\section{Method of Solution}

The initial conditions for the simulation of trajectories were obtained in the following manner. Assume that the plasma has a temperature $T$ associated with a Maxwellian velocity distribution. Taking the present normalization into account, the initial components of the velocity vector are obtained via

$$
v_{n 0, i}=\sqrt{\frac{2}{3}}\left(-2 \ln \left[R_{1 i}\right]\right)^{\frac{1}{2}} \cos \left(2 \pi R_{2 i}\right)
$$

with $i=x, y$, or $z$. Equation (12) represents random components of the initial velocity vector sampled from a Maxwellian distribution $[6,7]$, where $R_{j i}$ are all independent random numbers 
with a uniform distribution between zero and one. The initial conditions are obtained from the velocity vector $\boldsymbol{v}_{n 0}=v_{n 0, x} \hat{\boldsymbol{x}}+v_{n 0, y} \hat{\boldsymbol{y}}+v_{n 0, z} \hat{\boldsymbol{z}}$ and position vector $\boldsymbol{r}_{n 0}=R[-1,1] \hat{\boldsymbol{x}}-3 \hat{\boldsymbol{y}}+0 \hat{\boldsymbol{z}}$, where $\hat{\boldsymbol{x}}, \hat{\boldsymbol{y}}$, and $\hat{\boldsymbol{z}}$ are Cartesian unit vectors and $R\left[x_{1}, x_{2}\right]$ is a random number with a uniform distribution between $x_{1}$ and $x_{2}$. Note that the motion in the $y$ dimension takes charged particles towards [away from] the ASB when the velocity component in the $y$ dimension is positive [negative]; see Fig. 1. Consequently, the initial velocity component in the $y$ direction is calculated as prescribed by Eq. (12), and its absolute value is used so that all charged particles initially travel toward the ASB. The initial $x$ coordinate is sampled over two full periods of the simulation environment, which directly corresponds to the simulation region presented in Fig. 1. Additionally, two periods were chosen for the simulation region in order to allow for a large sampling of the phase-space but not so large that trajectories with glancing angles dominate the statistics obtained.

Taking advantage of the periodicity of the system, the electrostatic potential was computed for a region that is $0.5 S$ wide in the $x$ dimension and $5.0 S$ long in the $y$ dimension and then the solution was repeated in the $x$ dimension to complete the simulation region. The top of the simulation boundary corresponds to $y_{n}=1$ and the bottom to $y_{n}=-4$. The electrode labeled $V_{0}$ starts at $y_{n}=0$ and ends at $y_{n}=0.5$, the electrode labeled $V_{1}$ starts at $y_{n}=0.5$ and ends at $y_{n}=0.75$, and the electrode labeled $V_{2}$ starts at $y_{n}=0.75$ and ends at $y_{n}=1.0$, with one grid unit between adjacent electrodes. The electrostatic potential was computed using a finite differences sequential over-relaxation method [8]. In the calculation of the electrostatic potential, there are 40 grid units per period-lengths. Values were specified for applied normalized potentials $V_{n 0}, V_{n 1}$, and $V_{n 2}$ to establish the boundary conditions at the electrode locations. $V_{n 0}$ and $V_{n 2}$ were set to ground potential, $V_{n 0}=V_{n 2}=0$, whereas $V_{n 1}$ was biased to a positive value that was iteratively increased until a chosen value for $\phi_{n 0}$ was reached at the center of the anode gap. The electrostatic potential was obtained by first assigning values to the boundary regions where electrodes are located, then applying the finite difference sequential over-relaxation algorithm to the internal points, and assigning values to the remaining boundary points by requiring that the derivative normal to the boundary be zero. Such a procedure was carried out a sufficient number of times so that the difference in calculated normalized potential values from one iteration to the next was less than $1 \times 10^{-5}$ for all internal points.

The equations of motion were solved simultaneously via a "leap-frog" numerical approach 
(see, for example [9]). The time-step size was adjusted until the energy throughout the simulation was conserved to within $1 \%$ of the initial energy for all trajectories, during code development on a desktop computer. The same code was submitted for batch processing on a supercomputer. Some of the trajectories obtained from the supercomputer were also chosen and checked for energy conservation to within $1 \%$.

\section{RESULTS}

A parameter study for a single-species plasma can be performed in terms of the normalized parameters $\phi_{n 0}$ and $\delta$. Figure 2 was obtained by solving Eqs. (9)-(11) with $\phi_{n 0}=1$ and with $\delta=1000$ (1000 trajectories), $\delta=100$ (2000 trajectories) or $\delta=20$ (3000 trajectories) and by plotting the $x$ and $y$ components of the position vector in a parametric form. A different number of trajectories was chosen for the purpose of achieving the contrast in the figure. Figure 2 shows the general behavior of charged particle trajectories near an ASB as the inverse normalized Larmor radius $\delta$ is varied. Charged particle trajectories were also calculated in a similar manner but keeping the inverse normalized Larmor radius constant and varying the normalized electrostatic potential barrier $\phi_{n 0}$. The results are shown in Fig. 3.

The trends observed are (1) increasing the magnetic field sufficiently can effectively reflect charged particles away from most of the solid material and (2) increasing the electrostatic plugging sufficiently can reflect charged particles that would otherwise escape through the magnetic field cusps. The latter trend is, of course, directly affected by the kinetic energy of the charged particles, and those particles that escape lie in the high energy tail of the speed distribution. In the simulations, the trajectories were terminated when the particles

reached $y_{n} \geq 0.75$ (past the electrostatic potential barrier), or $y_{n} \leq-3$, or $\left|x_{n}\right| \geq 1$, or $\left|z_{n}\right| \geq 5$. However, Figs 2 and 3 only show a region defined by $y_{n} \leq 1, y_{n} \geq-1.5$, and $\left|x_{n}\right| \leq 1$ primarily to observe the modification of charged particle trajectories when charged particles approach the ASB. When a particle reached $y_{n} \geq 0.75$, its position vector was recorded. The $x$ component of the position vector for each of these particles was evaluated with respect to the center of the particular cusp that each of these entered and was assigned to a bin. There were 100 total bins for half of a spatial period in the simulation. Figures 4 and 5 show the data from 100,000 trajectories obtained by such a procedure. Figure 4 
presents the spatial profile of the particles that reached $y_{n} \approx 0.75$ for different values of the magnetic field/Larmor radius. Figure 5 is identical except that the magnetic field is fixed and the electrostatic potential barrier is varied. The solid curves in Figs. 4 and 5 are there to help distinguish the general trend that each of the data sets follow. The data series are labeled according to the parameter varied, and the corresponding percentage of particles that escaped confinement is shown. The plots show the most probable location through which particles can escape. Good confinement is defined here as the set of conditions that minimize the interaction of charged particles with the solid material. In the present study, magnetic confinement becomes apparent when the Larmor radius is smaller than 0.2 times the spatial period. The effect of electrostatic plugging is observed in Fig. 5. When the average thermal energy is less than the height of the electrostatic potential barrier, better confinement is achieved. For $\phi_{n 0}>5$ and $\delta>20$ the number of particles that escape confinement becomes negligible for the number of trajectories simulated in the present work.

\section{CONCLUSION}

An artificially structured boundary that produces electrostatically plugged magnetic field cusps has been presented as an alternative way to confine charged particles or plasma for ion source applications. Accumulation and confinement of highly pure or rare ions could benefit by decreased particle loss due to particle-solid material interaction. An ASB produces fields near the solid material boundary, and a nearly field free region exists away from the ASB. Charged particles can be can be confined by suitable adjustment of the applied electric and magnetic fields.

\section{ACKNOWLEDGEMENTS}

The authors would like to thank Allen S. Kiester for his help with code development. This material is based upon work supported by the Department of Energy under Grant No.DEFG02-06ER54883 and by the National Science Foundation under Grant No.PHY-1202428. 
Computational resources were provided by UNT's High Performance Computing Initiative.

[1] C. A. Ordonez, Journal of Applied Physics 106, 024905 (2009).

[2] J. L. Pacheco, C. A. Ordonez, and D. L. Weathers, IEEE Transactions on Plasma Science 39, $2424(2011)$.

[3] J. L. Pacheco, C. A. Ordonez, and D. L. Weathers, AIP Conference Proceedings 1525, 88 (2013).

[4] J. L. Pacheco, C. A. Ordonez, and D. L. Weathers, Physics of Plasmas 19, 102510 (2012).

[5] C. A. Ordonez, J. L. Pacheco, and D. L. Weathers, The Open Plasma Physics Journal 5, 1 (2012).

[6] C. A. Ordonez, W. D. Booth, R. Carrera, and M. E. Oakes, Radiation Effects and Defects in Solids 116, 37 (1991).

[7] G. E. P. Box and M. E. Muller, The Annals of Mathematical Statististics 29, 610 (1958).

[8] W. H. Press, B. P. Flannery, S. A. Teukolsky, and W. T. Vetterling, Numerical Recipes in C: The Art of Scientific Computing (Cambridge University Press, Cambridge. 1988).

[9] C. K. Birdsall and A. B. Langdon, Plasma Physics Via Computer Simulation (Institute of Physics Publishing, Philadelphia. 1991).

\section{FIGURES}




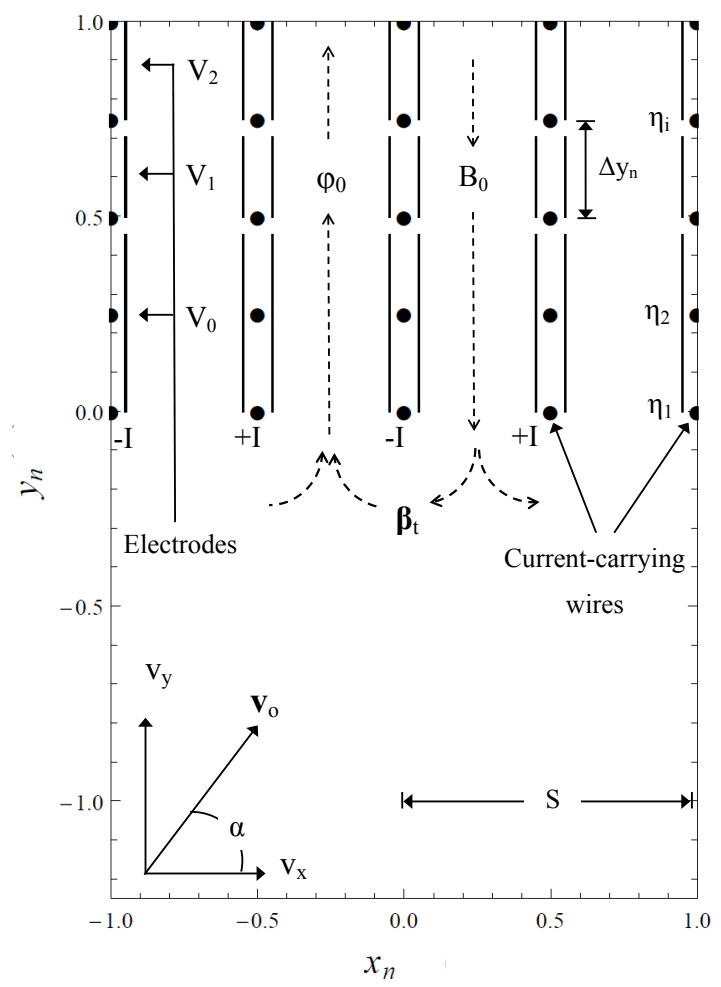

FIG. 1. Simulation environment representing two periods of a planar ASB. Ions are confined to the region below the ASB $\left(y_{n}<0\right)$. The lower edge of the ASB is located at $y_{n}=0$. The dots mark the positions of the current carrying wires, with current that alternates in sign from one column of wires to the next, $\pm I$. Magnetic field cusps are produced with the direction of the magnetic field labeled by $\boldsymbol{\beta}_{t}$. The electrodes are marked by lines, which represent their lengths and locations in the simulation environment. The current carrying wires and the electrodes are infinite in extent in the $z$ dimension. The electrostatic potential energy barrier is located in the region $0.5 \leq y_{n} \leq 0.75$, at the location of $V_{1} . V_{0}$ and $V_{2}$ are at ground potential. $\phi_{0}$ is the electric potential at the center of the anode gap, where the magnetic field has a magnitude $B_{0}$. See Eq. (2) for details regarding $\eta_{i}$, and $\Delta y_{n}$. 


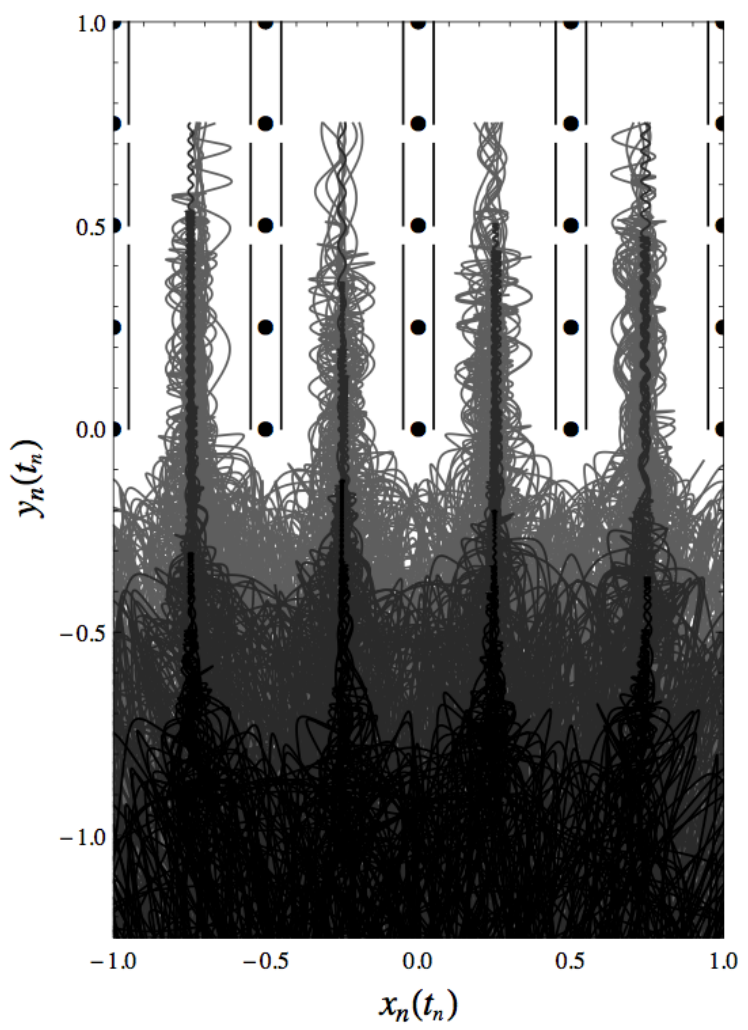

FIG. 2. Simulation that represents a two period segment of an ASB. The different shades show trajectories with $\phi_{n 0}=1$ and $\delta=1000$ (black), $\delta=100$ (dark gray), and $\delta=20$ (light gray). The trajectory calculation is terminated when a particle reaches $y_{n}=0.75$. 


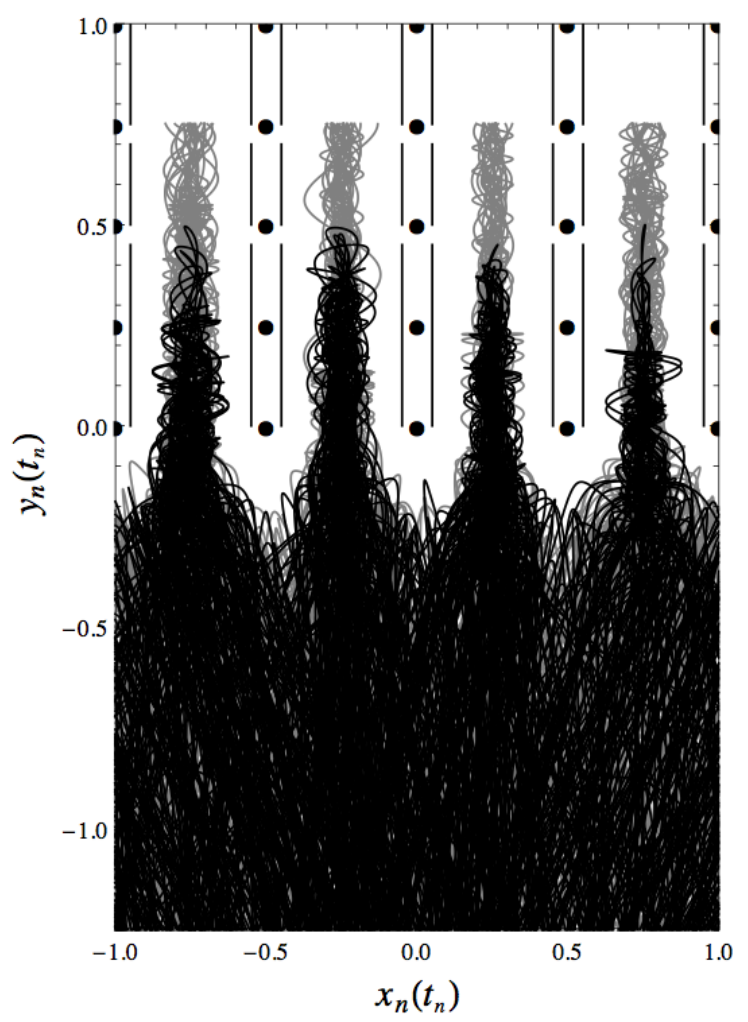

FIG. 3. Simulation that represents a two period segment of an ASB. The different shades show trajectories with $\delta=20$, and $\phi_{n 0}=0.5$ (light gray) and $\phi_{n 0}=5$ (black). The trajectory calculation is terminated when a particle reaches $y_{n}=0.75$.

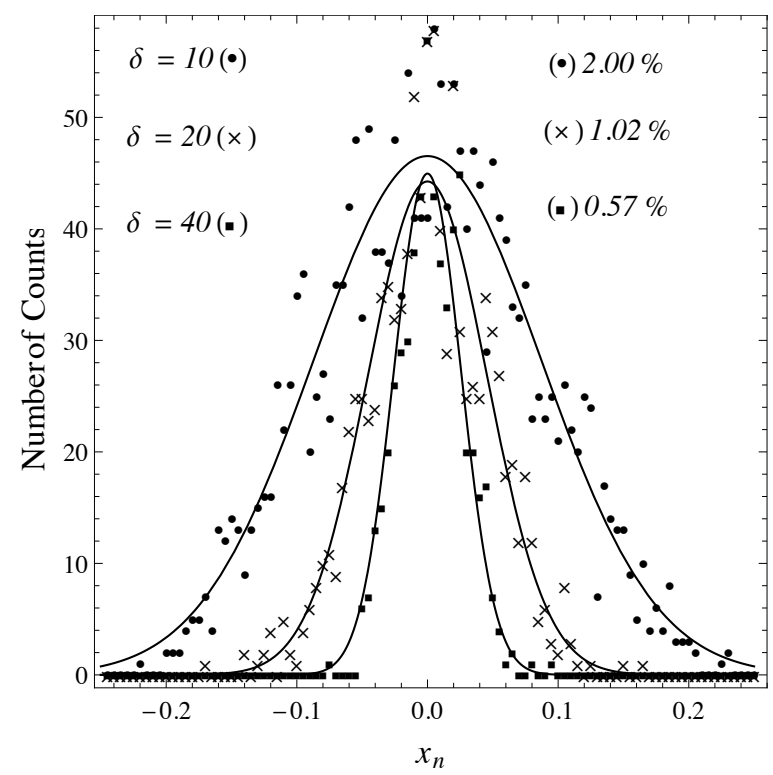


FIG. 4. Profile of the spatial distribution of charged particles that reached $y_{n} \geq 0.75$ after entering a cusp and overcoming the electrostatic potential barrier. The distribution of particles at $y_{n} \approx 0.75$ is for $\phi_{n 0}=1$ and $\delta=10,20$, and 40 . The data series are labeled according to the parameter varied, and the corresponding percentages of particles that reached $y_{n} \approx 0.75$ are indicated. The total number of trajectories simulated for each of these plots was 100,000.

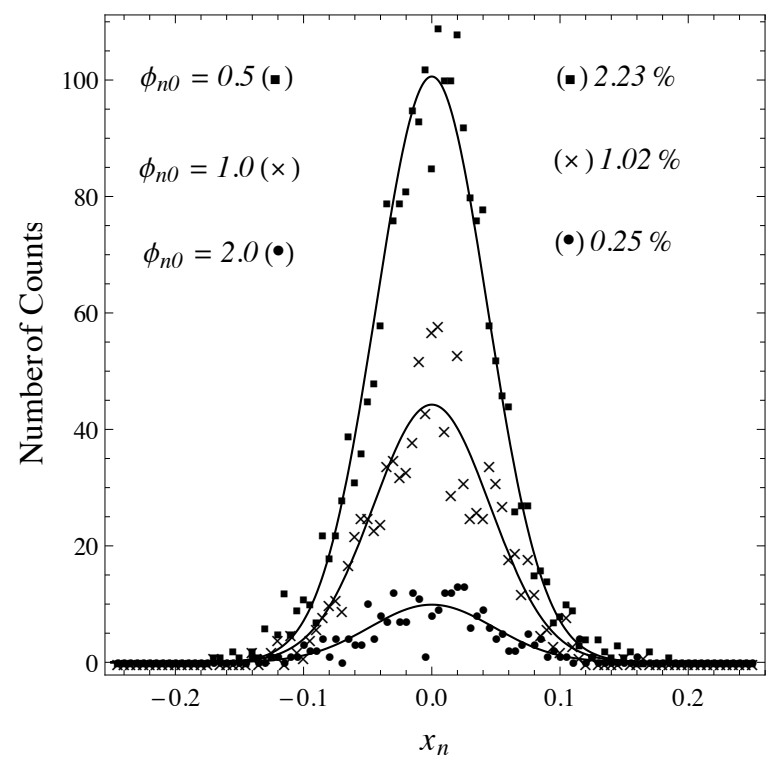

FIG. 5. Profile of the spatial distribution of charged particles that reached $y_{n} \geq 0.75$ after entering a cusp and overcoming the electrostatic potential barrier. The distribution is for $\delta=20$ and $\phi_{n 0}=$ $0.5,1$, and 2 . The data series are labeled according to the parameter varied, and the corresponding percentages of particles that reached $y_{n} \approx 0.75$ are indicated. The total number of trajectories simulated for each of these plots was 100,000. 
(C) 2020. Licensed under the Creative Commons Attribution (CC-BY-NC-ND) license (https://creativecommons.org/licenses/by-nc-nd/4.0/). 